\title{
Author Correction: Inflammasome signalling in brain function and neurodegenerative disease
}

\author{
Michael T. Heneka, Róisín M. McManus and Eicke LatziD
}

Nature Reviews Neuroscience (2018) https://doi.org/10.1038/s41583-018-0055-7

Published online 11 September 2018

In the originally published version of this article, the competing interests statement indicated that the authors had no competing interests; however, this statement was incorrect. The statement should have read as follows: "M.H. receives a consultation fee from IFM Therapeutics, LLC for consultations regarding the pathogenesis and interventional strategies of neurodegenerative disease. E.L. is a scientific co-founder and consultant to IFM Therapeutics. R.M.M. declares no competing interests.' This error has been corrected in the HTML and PDF versions of the article.

https://doi.org/10.1038/s41583-019-0137-1 I Published online 11 February 2019

\section{Author Correction: Epigenetic regulation in psychiatric disorders}

Nadia Tsankova, William Renthal, Arvind Kumar and Eric J. Nestler

Nature Reviews Neuroscience (2007) https://doi.org/10.1038/nrn2132

Published online 01 May 2007

In part c of Figure 1 in this article, the orientation of the tail of histone 3 with respect to the core region of this molecule was incorrect, and the amino acid residue $\mathrm{K} 79$ should not have been depicted in the tail. The corrected figure is shown below. The authors and editors thank T. Brown, R. Lober and C. Waker for bringing this error to our attention. Note that the present affiliation of the corresponding author and their current email address have been added to the correction notice.

https://doi.org/10.1038/s41583-018-0089-x I Published online 13 December 2018 